\title{
Periodic Solutions for Gause-Type Ratio-Dependent Predator-Prey Systems with Delays on Time Scales
}

\author{
Xiaoquan Ding, Hongyuan Liu, and Fengye Wang \\ School of Mathematics and Statistics, Henan University of Science and Technology, Luoyang, Henan 471023, China \\ Correspondence should be addressed to Xiaoquan Ding; xqding@haust.edu.cn
}

Received 28 February 2013; Accepted 27 May 2013

Academic Editor: Qiru Wang

Copyright (C) 2013 Xiaoquan Ding et al. This is an open access article distributed under the Creative Commons Attribution License, which permits unrestricted use, distribution, and reproduction in any medium, provided the original work is properly cited.

\begin{abstract}
This paper is devoted to periodic Gause-type ratio-dependent predator-prey systems with monotonic or nonmonotonic numerical responses on time scales. By using a continuation theorem based on coincidence degree theory, we establish easily verifiable criteria for the existence of periodic solutions. In particular, our results improve and generalize some known ones.
\end{abstract}

\section{Introduction}

In this paper, we consider the following periodic Gause-type ratio-dependent predator-prey system with time delays on a time scale $\mathbb{T}$ :

$$
\begin{aligned}
& u_{1}^{\Delta}(t)=f\left(t, \mathrm{e}^{u_{1}(t-\sigma(t))}\right)-p\left(t, \mathrm{e}^{u_{1}(t)-u_{2}(t)}\right), \\
& u_{2}^{\Delta}(t)=-d(t)+h\left(t, \mathrm{e}^{u_{1}(t-\tau(t))-u_{2}(t-\tau(t))}\right) .
\end{aligned}
$$

Here $\mathbb{T}$ is a periodic time scale which has the subspace topology inherited from the standard topology on $\mathbb{R}$. The symbol $\Delta$ stands for the delta-derivative which gives the ordinary derivative if $\mathbb{T}=\mathbb{R}$, and the forward difference operator if $\mathbb{T}=\mathbb{Z}$.

The theory of dynamic equations on time scales, which was introduced in order to unify differential and difference equations, has recently received a lot of attention, and many results on the issue have been documented in monographs $[1,2]$ and papers [3-12].

In system $(1)$, set $x(t)=\exp \left[u_{1}(t)\right], y(t)=\exp \left[u_{2}(t)\right]$. If $\mathbb{T}=\mathbb{R}$, then system (1) reduces to the standard Gausetype ratio-dependent predator-prey system governed by the ordinary differential equations

$$
\begin{aligned}
& x^{\prime}(t)=x(t) f(t, x(t-\sigma(t)))-y(t) g\left(t, \frac{x(t)}{y(t)}\right), \\
& y^{\prime}(t)=y(t)\left[-d(t)+h\left(t, \frac{x(t-\tau(t))}{y(t-\tau(t))}\right)\right],
\end{aligned}
$$

where $x(t)$ and $y(t)$ stand for the population of the prey and the predator, respectively. The function $f(t, v)$ is the growth rate of the prey in the absence of the predator. The function $d(t)$ is the death rate of the predator. The function $g(t, v)=: v p(t, v)$, called functional response of predator to prey, describes the change in the rate of exploitation of prey by a predator as a result of a change in the prey density. The function $h(t, v)$, called numerical response of predator to prey, describes the change in reproduction rate with changing prey density. If $\mathbb{T}=\mathbb{Z}$, then system (1) is reformulated as

$$
\begin{gathered}
x(k+1)=x(k) \exp \left[f(k, x(k-\sigma(k)))-p\left(k, \frac{x(k)}{y(k)}\right)\right], \\
y(k+1)=y(k) \exp \left[-d(k)+h\left(k, \frac{x(k-\tau(k))}{y(k-\tau(k))}\right)\right],
\end{gathered}
$$

which is the discrete time ratio-dependent predator-prey system and is a discrete analogue of (2).

We note that Ding [13], Ding and Jiang [14], Fan and Li [15], Fan et al. [16], Fan and Wang [17], Fan et al. [18], Hsu et al. [19], Kuang [20], Wang and Li [21], and Xia and Han [22] considered some special cases of system (2). Fan and Wang [23], and Xia et al. [24] discussed some special cases of system (3). Bohner et al. [4], and Shao [10] investigated some special cases of system (1). So far as we know, there is no published paper concerned system (1).

The main purpose of this paper is, by using the coincidence degree theory developed by Gaines and Mawhin [25], 
to derive sufficient conditions for the existence of periodic solutions of system (1). Furthermore, we will see that our results for the above system can be easily extended to the one with distributed or state-dependent delays. Our results improve or generalize some theorems in $[10,13-18,21,23]$.

\section{Preliminaries}

In this section, we briefly give some elements of the time scale calculus, recall the continuation theorem from coincidence degree theory, and state an auxiliary result that will be used in this paper.

First, let us present some foundational definitions and results from the calculus on time scales so that the paper is self-contained. For more details, we refer the reader to $[1,2]$.

A time scale $\mathbb{T}$ is an arbitrary nonempty closed subset $\mathbb{T}$ of the real numbers $\mathbb{R}$, which inherits the standard topology of $\mathbb{R}$. Thus, the real numbers $\mathbb{R}$, the integers $\mathbb{Z}$, and the natural numbers $\mathbb{N}$ are examples of time scales, while the rational numbers $\mathbb{Q}$ and the open interval $(1,2)$ are no time scales.

Let $\omega>0$. Throughout this paper, the time scale $\mathbb{T}$ is assumed to be $\omega$-periodic; that is, $t \in \mathbb{T}$ implies $t+\omega \in \mathbb{T}$. In particular, the time scale $\mathbb{T}$ under consideration is unbounded above and below.

For $t \in \mathbb{T}$, the forward and backward jump operators $\sigma, \rho$ : $\mathbb{T} \rightarrow \mathbb{R}$ are defined by

$$
\sigma(t)=\inf \{s \in \mathbb{T}: s>t\}, \quad \rho(t)=\sup \{s \in \mathbb{T}: s<t\},
$$

respectively.

If $\sigma(t)=t, t$ is called right-dense (otherwise: rightscattered), and if $\rho(t)=t$, then $t$ is called left-dense (otherwise: left-scattered).

A function $f: \mathbb{T} \rightarrow \mathbb{R}$ is said to be rd-continuous if it is continuous at right-dense points in $\mathbb{T}$ and its left-sided limits exist (finite) at left-dense points in $\mathbb{T}$. The set of rd-continuous functions is denoted by $C_{\mathrm{rd}}(\mathbb{T})$.

For $f: \mathbb{T} \rightarrow \mathbb{R}$ and $t \in \mathbb{T}$ we define $f^{\Delta}(t)$, the deltaderivative of $f$ at $t$, to be the number (provided it exists) with the property that, given any $\varepsilon>0$, there is a neighborhood $U$ of $t$ (i.e., $U=(t-\delta, t+\delta) \cap \mathbb{T}$ for some $\delta>0)$ in $\mathbb{t}$ such that

$$
\begin{gathered}
\left|[f(\sigma(t))-f(s)]-f^{\Delta}(t)[\sigma(t)-s]\right| \\
\quad \leq \varepsilon|\sigma(t)-s|, \quad \forall s \in U .
\end{gathered}
$$

$f$ is said to be delta-differentiable if its delta-derivative exists for all $t \in \mathbb{T}$. The set of functions $f: \mathbb{T} \rightarrow \mathbb{R}$ that are deltadifferentiable and whose delta-derivative are rd-continuous functions is denoted by $C_{\mathrm{rd}}^{1}(\mathbb{T})$.

A function $F: \mathbb{T} \rightarrow \mathbb{R}$ is called a delta-antiderivative of $f: \mathbb{T} \rightarrow \mathbb{R}$ provided $F^{\Delta}(t)=f(t)$, for all $t \in \mathbb{T}$. Then, we define the delta integral by

$$
\int_{a}^{b} f(t) \Delta t=F(b)-F(a), \quad \forall a, b \in \mathbb{T} .
$$

If $f$ is continuous, then $f$ is rd-continuous, and if $f$ is delta-differentiable at $t$, then $f$ is continuous at $t$. Moreover, every rd-continuous function on $\mathbb{T}$ has a delta-antiderivative.

Next, let us recall the continuation theorem in coincidence degree theory. To do so, we need to introduce the following notation.

Let $X, Y$ be real Banach spaces, $L:$ Dom $L \subset X \rightarrow Y$ be a linear mapping, and $N: X \rightarrow Y$ be a continuous mapping.

The mapping $L$ is said to be a Fredholm mapping of index zero, if $\operatorname{dim} \operatorname{Ker} L=\operatorname{codim} \operatorname{Im} L<+\infty$ and $\operatorname{Im} L$ is closed in $Y$.

If $L$ is a Fredholm mapping of index zero, then there exist continuous projectors $P: X \rightarrow X$ and $Q: Y \rightarrow Y$, such that $\operatorname{Im} P=\operatorname{Ker} L, \operatorname{Ker} Q=\operatorname{Im} L=\operatorname{Im}(I-Q)$. It follows that the restriction $L_{P}$ of $L$ to $\operatorname{Dom} L \cap \operatorname{Ker} P:(I-P) X \rightarrow \operatorname{Im} L$ is invertible. Denote the inverse of $L_{P}$ by $K_{P}$.

The mapping $N$ is said to be $L$-compact on $\bar{\Omega}$, if $\Omega$ is an open bounded subset of $X, Q N(\bar{\Omega})$ is bounded and $K_{P}(I-$ Q) $N: \bar{\Omega} \rightarrow X$ is compact.

Since $\operatorname{Im} Q$ is isomorphic to Ker $L$, there exists an isomorphism $J: \operatorname{Im} Q \rightarrow \operatorname{Ker} L$.

Here we state the Gaines-Mawhin theorem, which is a main tool in the proof of our main result.

Lemma 1 (Continuation Theorem [25, page 40]). Let $\Omega \subset X$ be an open bounded set, $L$ a Fredholm mapping of index zero, and $N$ L-compact on $\bar{\Omega}$. Assume
(a) for each $\lambda \in(0,1), x \in \partial \Omega \cap \operatorname{Dom} L, L x \neq \lambda N x$;
(b) for each $x \in \partial \Omega \cap \operatorname{Ker} L, Q N x \neq 0$;
(c) $\operatorname{deg}(J Q N, \Omega \cap \operatorname{Ker} L, 0) \neq 0$.

\section{Then $L x=N x$ has at least one solution in $\bar{\Omega} \cap \operatorname{Dom} L$.}

For convenience and simplicity in the following discussion, we always use the following notation:

$$
\begin{gathered}
\kappa=\min \{[0,+\infty) \cap \mathbb{T}\}, \quad I_{\omega}=[\kappa, \kappa+\omega] \cap \mathbb{T}, \\
\widehat{a}=\frac{1}{\omega} \int_{\kappa}^{\kappa+\omega} a(t) \Delta t, \quad \widehat{A}=\frac{1}{\omega} \int_{\kappa}^{\kappa+\omega}|a(t)| \Delta t, \\
\widetilde{b}(x)=\frac{1}{\omega} \int_{\kappa}^{\kappa+\omega} b(t, x) \Delta t, \\
\widetilde{B}(x)=\frac{1}{\omega} \int_{\kappa}^{\kappa+\omega}|b(t, x)| \Delta t,
\end{gathered}
$$

where $a \in C_{\mathrm{rd}}(\mathbb{T})$ is an $\omega$-periodic function, $b: \mathbb{T} \times \mathbb{R} \rightarrow \mathbb{R}$ is rd-continuous and $\omega$-periodic in its first variable.

In order to achieve the prior estimation in the case of dynamic equations on a time scale $\mathbb{T}$, we also require the following inequality which is proved in [11, Theorem 2.1]. 
Lemma 2. Let $t_{1}, t_{2} \in I_{\omega}$, and $t \in \mathbb{T}$. If $\varphi \in C_{\mathrm{rd}}^{1}(\mathbb{T})$ is an $\omega$-periodic real function, then

$$
\begin{aligned}
& \varphi(t) \leq \varphi\left(t_{1}\right)+\frac{1}{2} \int_{\kappa}^{\kappa+\omega}\left|\varphi^{\Delta}(t)\right| \Delta t, \\
& \varphi(t) \geq \varphi\left(t_{2}\right)-\frac{1}{2} \int_{\kappa}^{\kappa+\omega}\left|\varphi^{\Delta}(t)\right| \Delta t .
\end{aligned}
$$

\section{Existence of Periodic Solutions}

In this section, we study the existence of periodic solutions of system (1). For the sake of generality, we make the following fundamental assumptions for system (1):

$\left(\mathrm{H}_{1}\right) \sigma, \tau: \mathbb{T} \rightarrow \mathbb{R}^{+}$are $\mathrm{rd}$-continuous and $\omega$-periodic such that $t-\sigma(t), t-\tau(t) \in \mathbb{T}$ for all $t \in \mathbb{T}$.

$\left(\mathrm{H}_{2}\right) d: \mathbb{T} \rightarrow \mathbb{R}$ is rd-continuous and $\omega$-periodic such that $\widehat{d}>0$.

$\left(\mathrm{H}_{3}\right) f: \mathbb{T} \times \mathbb{R} \rightarrow \mathbb{R}$ is rd-continuous and $\omega$-periodic in the first variable and is continuously differentiable in the second variable such that $\tilde{f}(0)>0, \lim _{x \rightarrow+\infty} \tilde{f}(x)<$ 0 , and $(\partial f / \partial x)(t, x)<0$ for all $t \in \mathbb{T}, x>0$.

$\left(\mathrm{H}_{4}\right) p: \mathbb{T} \times \mathbb{R} \rightarrow \mathbb{R}^{+}$is rd-continuous and $\omega$-periodic in the first variable and is continuously differentiable in the second variable.

$\left(\mathrm{H}_{5}\right) h: \mathbb{T} \times \mathbb{R} \rightarrow \mathbb{R}^{+}$is rd-continuous and $\omega$-periodic in the first variable and is continuously differentiable in the second variable such that $h(t, 0)=0$ for all $t \in \mathbb{T}$.

$\left(\mathrm{H}_{6}\right) \tilde{f}(0)>\widehat{c}$ and $\sup _{x \geq 0} \tilde{h}(x)>\widehat{d}$, where $c(t)=$ : $\sup _{x>0} p(t, x)$ for all $t \in \mathbb{T}$.

From $\left(\mathrm{H}_{3}\right)$, we have

$$
\tilde{f}^{\prime}(x)=\frac{1}{\omega} \int_{\kappa}^{\kappa+\omega} \frac{\partial f}{\partial x}(t, x) \Delta t<0 .
$$

Thus $\tilde{f}(x)$ is strictly decreasing on $[0,+\infty)$. From this, $\left(\mathrm{H}_{3}\right)$, and $\left(\mathrm{H}_{6}\right)$, one can easily see that each of the equations

$$
\tilde{f}(x)=\frac{1}{\omega} \int_{\kappa}^{\kappa+\omega} \sup _{x>0} p(t, x) \Delta t, \quad \tilde{f}(x)=0,
$$

has a unique positive solution. Let $V_{1}, V_{2}$ be their solutions, respectively, then $V_{1}<V_{2}$.

3.1. Monotone Case. In this section, we study the existence of $\omega$-periodic solution of system (1) when the numerical response function $h(t, x)$ satisfies the following monotone condition:

$\left(\mathrm{H}_{7}\right)(\partial h / \partial x)(t, x)>0$ for $t \in \mathbb{T}, x>0$.

From $\left(\mathrm{H}_{5}\right)$ and $\left(\mathrm{H}_{7}\right)$, we have

$$
\tilde{h}^{\prime}(x)=\frac{1}{\omega} \int_{\kappa}^{\kappa+\omega} \frac{\partial h}{\partial x}(t, x) \Delta t>0 .
$$

Then $\tilde{h}(x)$ is strictly increasing on $[0,+\infty)$. From this, $\left(\mathrm{H}_{5}\right)$, and $\left(\mathrm{H}_{6}\right)$, one can easily see that the equation $\widetilde{h}(x)=\widehat{d}$ has a unique positive solution. Let $v_{0}$ be its solution.
Theorem 3. Suppose that $\left(H_{1}\right)-\left(H_{7}\right)$ hold. Then system (1) has at least one $\omega$-periodic solution.

Proof. Take

$$
\begin{gathered}
X=Y=\left\{u=\left(u_{1}(t), u_{2}(t)\right)^{\mathrm{T}} \mid u_{i} \in C(\mathbb{T}),\right. \\
\left.u_{i}(t+\omega)=u_{i}(t), i=1,2\right\}, \\
\|u\|=\left\|\left(u_{1}(t), u_{2}(t)\right)^{\mathrm{T}}\right\|=\max _{t \in I_{\omega}}\left|u_{1}(t)\right|+\max _{t \in I_{\omega}}\left|u_{2}(t)\right|,
\end{gathered}
$$

then $X$ and $Y$ are Banach spaces with the norm $\|\cdot\|$. Set

$$
L: \operatorname{Dom} L \subset X \longrightarrow Y, \quad L\left(\begin{array}{l}
u_{1}(t) \\
u_{2}(t)
\end{array}\right)=\left(\begin{array}{l}
u_{1}^{\Delta}(t) \\
u_{2}^{\Delta}(t)
\end{array}\right)
$$

where $\operatorname{Dom} L=\left\{u=\left(u_{1}(t), u_{2}(t)\right)^{\mathrm{T}} \in X \mid u_{i} \in C_{\mathrm{rd}}^{1}(\mathbb{T}), i=\right.$ $1,2\}$ and

$$
\begin{gathered}
N: X \rightarrow Y, \\
N\left(\begin{array}{l}
u_{1}(t) \\
u_{2}(t)
\end{array}\right)=\left(\begin{array}{c}
f\left(t, \mathrm{e}^{u_{1}(t-\sigma(t))}\right)-p\left(t, \mathrm{e}^{u_{1}(t)-u_{2}(t)}\right) \\
-d(t)+h\left(t, \mathrm{e}^{u_{1}(t-\tau(t))-u_{2}(t-\tau(t))}\right)
\end{array}\right) .
\end{gathered}
$$

With these notations system (1) can be written in the form

$$
L u=N u, \quad u \in X
$$

Obviously, $\operatorname{Ker} L=\mathbb{R}^{2}, \operatorname{Im} L=\left\{\left(u_{1}(t), u_{2}(t)\right)^{\mathrm{T}} \in Y: \widehat{u}_{i}=\right.$ $0, i=1,2\}$ is closed in $Y$, and $\operatorname{dimKer} L=\operatorname{codim} \operatorname{Im} L=2$. Therefore $L$ is a Fredholm mapping of index zero. Now define two projectors $P: X \rightarrow X$ and $Q: Y \rightarrow Y$ as

$$
P\left(\begin{array}{l}
u_{1}(t) \\
u_{2}(t)
\end{array}\right)=Q\left(\begin{array}{l}
u_{1}(t) \\
u_{2}(t)
\end{array}\right)=\left(\begin{array}{l}
\widehat{u}_{1} \\
\widehat{u}_{2}
\end{array}\right), \quad\left(\begin{array}{l}
u_{1}(t) \\
u_{2}(t)
\end{array}\right) \in X=Y
$$

then $P$ and $Q$ are continuous projectors such that

$$
\operatorname{Im} P=\operatorname{Ker} L, \quad \operatorname{Ker} Q=\operatorname{Im} L=\operatorname{Im}(I-Q) .
$$

Furthermore, through an easy computation we find that the generalized inverse $K_{P}$ of $L_{P}$ has the form

$$
\begin{gathered}
K_{P}: \operatorname{Im} L \longrightarrow \operatorname{Dom} L \cap \operatorname{Ker} P \\
K_{P}(u)=\int_{\kappa}^{t} u(s) \Delta s-\frac{1}{\omega} \int_{\kappa}^{\kappa+\omega} \int_{\kappa}^{t} u(s) \Delta s \Delta t .
\end{gathered}
$$


Then $Q N: X \rightarrow Y$ and $K_{P}(I-Q) N: X \rightarrow X$ read as

$$
\begin{aligned}
& \text { QNu } \\
& =\frac{1}{\omega}\left(\begin{array}{c}
\int_{\kappa}^{\kappa+\omega}\left[f\left(t, \mathrm{e}^{u_{1}(t-\sigma(t))}\right)-p\left(t, \mathrm{e}^{u_{1}(t)-u_{2}(t)}\right)\right] \Delta t \\
\int_{\kappa}^{\kappa+\omega}\left[-d(t)+h\left(t, \mathrm{e}^{u_{1}(t-\tau(t))-u_{2}(t-\tau(t))}\right)\right] \Delta t
\end{array}\right), \\
& K_{P}(I-Q) N u \\
& =\int_{\kappa}^{t} N u(s) \Delta s-\frac{1}{\omega} \int_{\kappa}^{\kappa+\omega} \int_{\kappa}^{t} N u(s) \Delta s \Delta t \\
& -\frac{1}{\omega}\left(t-\kappa-\frac{1}{\omega} \int_{\kappa}^{\kappa+\omega}(s-\kappa) \Delta s\right) \int_{\kappa}^{\kappa+\omega} N u(s) \Delta s .
\end{aligned}
$$

Clearly, $Q N$ and $K_{P}(I-Q) N$ are continuous. By using the Arzela-Ascoli theorem, it is not difficult to prove that $\overline{K_{P}(I-Q) N(\bar{\Omega})}$ is compact for any open bounded set $\Omega \subset X$. Moreover, $Q N(\bar{\Omega})$ is bounded. Therefore $N$ is $L$-compact on $\bar{\Omega}$ with any open bounded set $\Omega \subset X$.

In order to apply Lemma 1 , we need to find an appropriate open, bounded subset in $X$. Corresponding to the operator equation $L u=\lambda N u, \lambda \in(0,1)$, we have

$$
\begin{aligned}
& u_{1}^{\Delta}(t)=\lambda\left[f\left(t, \mathrm{e}^{u_{1}(t-\sigma(t))}\right)-p\left(t, \mathrm{e}^{u_{1}(t)-u_{2}(t)}\right)\right], \\
& u_{2}^{\Delta}(t)=\lambda\left[-d(t)+h\left(t, \mathrm{e}^{u_{1}(t-\tau(t))-u_{2}(t-\tau(t))}\right)\right] .
\end{aligned}
$$

Suppose that $\left(u_{1}(t), u_{2}(t)\right)^{\mathrm{T}} \in X$ is a solution of (20) for a certain $\lambda \in(0,1)$. Integrating (20) on both sides from $\kappa$ to $\kappa+\omega$ leads to

$$
\begin{gathered}
\int_{\kappa}^{\kappa+\omega} \lambda\left[f\left(t, \mathrm{e}^{u_{1}(t-\sigma(t))}\right)-p\left(t, \mathrm{e}^{u_{1}(t)-u_{2}(t)}\right)\right] \Delta t=0, \\
\int_{\kappa}^{\kappa+\omega} \lambda\left[-d(t)+h\left(t, \mathrm{e}^{u_{1}(t-\tau(t))-u_{2}(t-\tau(t))}\right)\right] \Delta t=0 .
\end{gathered}
$$

That is

$$
\begin{aligned}
& \int_{\kappa}^{\kappa+\omega} f\left(t, \mathrm{e}^{u_{1}(t-\sigma(t))}\right) \Delta t=\int_{\kappa}^{\kappa+\omega} p\left(t, \mathrm{e}^{u_{1}(t)-u_{2}(t)}\right) \Delta t, \\
& \int_{\kappa}^{\kappa+\omega} h\left(t, \mathrm{e}^{u_{1}(t-\tau(t))-u_{2}(t-\tau(t))}\right) \Delta t=\int_{\kappa}^{\kappa+\omega} d(t) \Delta t=\widehat{d} \omega .
\end{aligned}
$$

From (22), we have

$$
\begin{array}{r}
\tilde{f}(0) \omega=\int_{\kappa}^{\kappa+\omega}\left[f(t, 0)-f\left(t, \mathrm{e}^{u_{1}(t-\sigma(t))}\right)\right. \\
\left.+p\left(t, \mathrm{e}^{u_{1}(t)-u_{2}(t)}\right)\right] \Delta t .
\end{array}
$$

It follows from (20), (22), (23), (24), and $\left(\mathrm{H}_{3}\right)-\left(\mathrm{H}_{5}\right)$ that

$$
\begin{aligned}
& \int_{\kappa}^{\kappa+\omega}\left|u_{1}^{\Delta}(t)\right| \Delta t \\
& \leq \lambda \int_{\kappa}^{\kappa+\omega}\left|f\left(t, \mathrm{e}^{u_{1}(t-\sigma(t))}\right)-m\left(t, \mathrm{e}^{u_{1}(t)-u_{2}(t)}\right)\right| \Delta t \\
& <\int_{\kappa}^{\kappa+\omega}\left\{\left[f(t, 0)-f\left(t, \mathrm{e}^{u_{1}(t-\sigma(t))}\right)\right]+p\left(t, \mathrm{e}^{u_{1}(t)-u_{2}(t)}\right)\right\} \Delta t \\
& \quad+\int_{\kappa}^{\kappa+\omega}|f(t, 0)| \Delta t=[\widetilde{F}(0)+\widetilde{f}(0)] \omega \\
& \int_{\kappa}^{\kappa+\omega}\left|u_{2}^{\Delta}(t)\right| \Delta t \\
& \leq \lambda \int_{\kappa}^{\kappa+\omega}\left|-d(t)+h\left(t, \mathrm{e}^{u_{1}(t-\tau(t))-u_{2}(t-\tau(t))}\right)\right| \Delta t \\
& <\int_{\kappa}^{\kappa+\omega}|d(t)| \Delta t+\int_{\kappa}^{\kappa+\omega} h\left(t, \mathrm{e}^{u_{1}(t-\tau(t))-u_{2}(t-\tau(t))}\right) \Delta t \\
& =(\widehat{D}+\widehat{d}) \omega .
\end{aligned}
$$

Since $\left(u_{1}(t), u_{2}(t)\right)^{\mathrm{T}} \in X$, there exist $\xi_{i}, \eta_{i} \in I_{\omega}$ such that

$$
u_{i}\left(\xi_{i}\right)=\max _{t \in I_{\omega}} u_{i}(t), \quad u_{i}\left(\eta_{i}\right)=\min _{t \in I_{\omega}} u_{\mathrm{i}}(t), \quad i=1,2 .
$$

Then from (22), (27), $\left(\mathrm{H}_{3}\right),\left(\mathrm{H}_{4}\right)$, and the monotonicity of function $f$, we have

$$
\begin{aligned}
\tilde{f}\left(\mathrm{e}^{u_{1}\left(\xi_{1}\right)}\right) & =\frac{1}{\omega} \int_{\kappa}^{\kappa+\omega} f\left(t, \mathrm{e}^{u_{1}\left(\xi_{1}\right)}\right) \Delta t \\
& \leq \frac{1}{\omega} \int_{\kappa}^{\kappa+\omega} f\left(t, \mathrm{e}^{u_{1}(t-\sigma(t))}\right) \Delta t \\
& \leq \frac{1}{\omega} \int_{\kappa}^{\kappa+\omega} \sup _{x>0} p(t, x) \Delta t,
\end{aligned}
$$

which, together with the monotonicity of function $\tilde{f}$, leads to

$$
u_{1}\left(\xi_{1}\right) \geq \ln V_{1} \text {. }
$$

By Lemma 2, we obtain from (25) and (29) that for all $t \in I_{\omega}$

$$
\begin{aligned}
u_{1}(t) & \geq u_{1}\left(\xi_{1}\right)-\frac{1}{2} \int_{\kappa}^{\kappa+\omega}\left|u_{1}^{\Delta}(t)\right| \Delta t \\
& >\ln V_{1}-\frac{\widetilde{F}(0)+\widetilde{f}(0)}{2} \omega:=B_{1} .
\end{aligned}
$$

From (22), (27), $\left(\mathrm{H}_{3}\right)$, and the monotonicity of function $f$, we have

$$
\begin{aligned}
\tilde{f}\left(\mathrm{e}^{u_{1}\left(\eta_{1}\right)}\right) & =\frac{1}{\omega} \int_{\kappa}^{\kappa+\omega} f\left(t, \mathrm{e}^{u_{1}\left(\eta_{1}\right)}\right) \Delta t \\
& \geq \frac{1}{\omega} \int_{\kappa}^{\kappa+\omega} f\left(t, \mathrm{e}^{u_{1}(t-\sigma(t))}\right) \Delta t>0,
\end{aligned}
$$


which, together with the monotonicity of function $\widetilde{f}$, leads to

$$
u_{1}\left(\eta_{1}\right) \leq \ln V_{2}
$$

By Lemma 2, we get from (25) and (32) that for all $t \in I_{\omega}$

$$
\begin{aligned}
u_{1}(t) & \leq u_{1}\left(\eta_{1}\right)+\frac{1}{2} \int_{\kappa}^{\kappa+\omega}\left|u_{1}^{\Delta}(t)\right| \Delta t \\
& <\ln V_{2}+\frac{\widetilde{F}(0)+\widetilde{f}(0)}{2} \omega:=B_{2} .
\end{aligned}
$$

From (23), (27), $\left(\mathrm{H}_{5}\right)$, and the monotonicity of function $h$, we have

$$
\begin{aligned}
& \tilde{h}\left(\mathrm{e}^{u_{1}\left(\xi_{1}\right)-u_{2}\left(\eta_{2}\right)}\right) \geq \frac{1}{\omega} \int_{\kappa}^{\kappa+\omega} h\left(t, \mathrm{e}^{u_{1}(t-\tau(t))-u_{2}(t-\tau(t))}\right) \Delta t=\widehat{d}, \\
& \widetilde{h}\left(\mathrm{e}^{u_{1}\left(\eta_{1}\right)-u_{2}\left(\xi_{2}\right)}\right) \leq \frac{1}{\omega} \int_{\kappa}^{\kappa+\omega} h\left(t, \mathrm{e}^{u_{1}(t-\tau(t))-u_{2}(t-\tau(t))}\right) \Delta t=\widehat{d},
\end{aligned}
$$

which, together with the monotonicity of function $\widetilde{h}$, lead to

$$
\mathrm{e}^{u_{1}\left(\xi_{1}\right)-u_{2}\left(\eta_{2}\right)} \geq v_{0}, \quad \mathrm{e}^{u_{1}\left(\eta_{1}\right)-u_{2}\left(\xi_{2}\right)} \leq v_{0} .
$$

That is

$$
\begin{aligned}
& u_{2}\left(\eta_{2}\right) \leq u_{1}\left(\xi_{1}\right)-\ln v_{0} \\
& u_{2}\left(\xi_{2}\right) \geq u_{1}\left(\eta_{1}\right)-\ln v_{0} .
\end{aligned}
$$

By Lemma 2, we get from (26), (30), (33), and (36) that for all $t \in I_{\omega}$

$$
\begin{aligned}
u_{2}(t) & \leq u_{2}\left(\eta_{2}\right)+\frac{1}{2} \int_{\kappa}^{\kappa+\omega}\left|u_{2}^{\Delta}(t)\right| \Delta t \\
& <B_{1}-\ln v_{0}+\frac{\widehat{D}+\widehat{d}}{2} \omega:=B_{3}, \\
u_{2}(t) & \geq u_{2}\left(\xi_{2}\right)-\frac{1}{2} \int_{\kappa}^{\kappa+\omega}\left|u_{2}^{\Delta}(t)\right| \Delta t \\
& >B_{2}-\ln v_{0}-\frac{\widehat{D}+\widehat{d}}{2} \omega:=B_{4} .
\end{aligned}
$$

In view of (30), (33), (37), we obtain

$$
\begin{aligned}
& \max _{t \in I_{\omega}}\left|u_{1}(t)\right| \leq \max \left\{\left|B_{1}\right|,\left|B_{2}\right|\right\}:=B_{5}, \\
& \max _{t \in I_{\omega}}\left|u_{2}(t)\right| \leq \max \left\{\left|B_{3}\right|,\left|B_{4}\right|\right\}:=B_{6} .
\end{aligned}
$$

Clearly, $B_{5}$ and $B_{6}$ are independent of $\lambda$.

From $\left(\mathrm{H}_{3}\right),\left(\mathrm{H}_{6}\right)$, and the monotonicity of functions $\tilde{f}$ and $\widetilde{h}$, it is easy to show that the algebraic system

$$
\begin{gathered}
\tilde{f}\left(\mathrm{e}^{u_{1}}\right)-\widetilde{p}\left(\mathrm{e}^{u_{1}-u_{2}}\right)=0, \\
-\widehat{d}+\widetilde{h}\left(\mathrm{e}^{u_{1}-u_{2}}\right)=0,
\end{gathered}
$$

has a unique solution $\left(u_{1}^{*}, u_{2}^{*}\right)^{\mathrm{T}} \in \mathbb{R}^{2}$.
We now take $\Omega=\left\{\left(u_{1}(t), u_{2}(t)\right)^{\mathrm{T}} \in X: \|\left(u_{1}\right.\right.$ $\left.\left.(t), u_{2}(t)\right)^{\mathrm{T}} \|<B_{5}+B_{6}+B_{0}\right\}$, where $B_{0}$ is taken sufficiently large such that $\left\|\left(u_{1}^{*}, u_{2}^{*}\right)^{\mathrm{T}}\right\|=\left|u_{1}^{*}\right|+\left|u_{2}^{*}\right|<B_{0}$. With the help of (38) and (39), it is easy to see that $\Omega$ satisfies condition (a) in Lemma 1. When $\left(u_{1}(t), u_{2}(t)\right)^{\mathrm{T}} \in \partial \Omega \cap \operatorname{Ker} L=\partial \Omega \cap \mathbb{R}^{2}$, $\left(u_{1}(t), u_{2}(t)\right)^{\mathrm{T}}$ is a constant vector in $\mathbb{R}^{2}$ with $\left|u_{1}\right|+\left|u_{2}\right|=$ $B_{5}+B_{6}+B_{0}$. Thus, we have

$$
Q N\left(\begin{array}{l}
u_{1} \\
u_{2}
\end{array}\right)=\left(\begin{array}{c}
\tilde{f}\left(\mathrm{e}^{u_{1}}\right)-\tilde{p}\left(\mathrm{e}^{u_{1}-u_{2}}\right) \\
-\widehat{d}+\widetilde{h}\left(\mathrm{e}^{u_{1}-u_{2}}\right)
\end{array}\right) \neq\left(\begin{array}{l}
0 \\
0
\end{array}\right) .
$$

This proves that condition (b) in Lemma 1 is satisfied.

Taking $J=I: \operatorname{Im} Q \rightarrow \operatorname{Ker} L,\left(u_{1}, u_{2}\right)^{\mathrm{T}} \rightarrow\left(u_{1}, u_{2}\right)^{\mathrm{T}}$, a direct calculation shows that

$$
\begin{aligned}
\operatorname{deg}( & (T N, \Omega \cap \operatorname{Ker} L, 0) \\
& =\operatorname{sign}\left\{-\tilde{f}^{\prime}\left(\mathrm{e}^{u_{1}^{*}}\right) \tilde{h}^{\prime}\left(\mathrm{e}^{u_{1}^{*}-u_{2}^{*}}\right) \mathrm{e}^{2 u_{1}^{*}-u_{2}^{*}}\right\} \\
& =1 \neq 0 .
\end{aligned}
$$

By now we have proved that $\Omega$ satisfies all the requirements in Lemma 1 . Hence, (1) has at least one $\omega$-periodic solution. This completes the proof.

Noticing that both systems (2) and (3) are special cases of system (1), by Theorem 3, we can obtain the following results.

Theorem 4. Suppose that $\left(H_{1}\right)-\left(H_{7}\right)$ hold. Then system (2) has at least one positive $\omega$-periodic solution.

Theorem 5. Suppose that $\left(H_{1}\right)-\left(H_{7}\right)$ hold. Then system (3) has at least one positive $\omega$-periodic solution.

3.2. Nonmonotone Case. In this section, we study the existence of $\omega$-periodic solution of system (1) when the numerical response function $h(t, x)$ satisfies the following nonmonotone condition:

$\left(\mathrm{H}_{8}\right) \lim _{v \rightarrow+\infty} h(t, x)=0$ for $t \in \mathbb{R}$, and there exists a positive constant $\underline{v}$ such that

$$
(x-\underline{v}) \frac{\partial h}{\partial x}(t, x)<0, \quad \text { for } t \in \mathbb{T}, x>0, x \neq \underline{v} .
$$

By $\left(\mathrm{H}_{5}\right)$ and $\left(\mathrm{H}_{8}\right)$, we have

$$
\begin{gathered}
(x-\underline{v}) \tilde{h}^{\prime}(x)=\frac{1}{\omega} \int_{\kappa}^{\kappa+\omega}(x-\underline{v}) \frac{\partial h}{\partial x}(t, x) \Delta t<0, \\
\lim _{v \rightarrow+\infty} \tilde{h}(x)=\frac{1}{\omega} \int_{\kappa}^{\kappa+\omega} \lim _{x \rightarrow+\infty} h(t, x) \Delta t=0,
\end{gathered}
$$

then $\widetilde{h}(x)$ is strictly increasing on $[0, \underline{v}]$ and strictly decreasing on $[\underline{v},+\infty)$. By these, $\left(\mathrm{H}_{5}\right)$ and $\left(\mathrm{H}_{6}\right)$, one can easily see that the equation

$$
\widetilde{h}(x)=\widehat{d}
$$

has two distinct positive solutions, namely, $v_{1}, v_{2}$. Without loss of generality, we suppose that $v_{1}<v_{2}$, then $v_{1}<\underline{v}<v_{2}$. 
Theorem 6. In addition to $\left(H_{1}\right)-\left(H_{6}\right)$ and $\left(H_{8}\right)$, suppose further that the following condition holds:

$\left(\mathrm{H}_{9}\right)\left(V_{2} / V_{1}\right) e^{[\tilde{F}(0)+\tilde{f}(0)+\widehat{D}+\widehat{d}] \omega}<v_{2} / v_{1}$.

Then system (1) has at least two $\omega$-periodic solutions.

Proof. The proof is similar to that of Theorem 3. To complete the proof, we need to find two disjoint open bounded subsets in $X$. Under condition $\left(\mathrm{H}_{9}\right),(34)$ is no longer valid. So, we need to make a corresponding change.

Designating $z(t)=u_{1}(t)-u_{2}(t)$, there also exist $\xi_{0}, \eta_{0} \in I_{\omega}$ such that

$$
z\left(\xi_{0}\right)=\max _{t \in I_{\omega}} z(t), \quad z\left(\eta_{0}\right)=\min _{t \in I_{\omega}} z(t) .
$$

From (23), (27), and the monotonicity of functions $h$ and $\widehat{h}$, we will show that $z\left(\xi_{0}\right)$ and $z\left(\eta_{0}\right)$ can not simultaneously lie in $\left(-\infty, \ln v_{1}\right),\left(\ln v_{1}, \ln v_{2}\right)$ or $\left(\ln v_{2},+\infty\right)$. In fact, if $z\left(\eta_{0}\right) \leq$ $z\left(\xi_{0}\right)<\ln v_{1}$, then

$$
\begin{aligned}
\widehat{d} & =\frac{1}{\omega} \int_{\kappa}^{\kappa+\omega} h\left(t, \mathrm{e}^{z(t-\tau(t))}\right) \Delta t \\
& \leq \widetilde{h}\left(\mathrm{e}^{z\left(\xi_{0}\right)}\right)<\widetilde{h}\left(v_{1}\right)=\widehat{d} .
\end{aligned}
$$

This is a contradiction. If $\ln v_{2}<z\left(\eta_{0}\right) \leq z\left(\xi_{0}\right)$, then

$$
\begin{aligned}
\widehat{d} & =\frac{1}{\omega} \int_{\kappa}^{\kappa+\omega} h\left(t, \mathrm{e}^{z(t-\tau(t))}\right) \Delta t \\
& \leq \widetilde{h}\left(\mathrm{e}^{z\left(\eta_{0}\right)}\right)<\widetilde{h}\left(v_{2}\right)=\widehat{d} .
\end{aligned}
$$

This is also a contradiction. If $\ln v_{1}<z\left(\eta_{0}\right) \leq z\left(\xi_{0}\right)<\ln v_{2}$, then

$$
\begin{aligned}
\widehat{d} & =\frac{1}{\omega} \int_{\kappa}^{\kappa+\omega} h\left(t, \mathrm{e}^{z(t-\tau(t))}\right) \Delta t \\
& \geq \min \left\{\widetilde{h}\left(\mathrm{e}^{z\left(\xi_{0}\right)}\right), \widetilde{h}\left(\mathrm{e}^{z\left(\eta_{0}\right)}\right)\right\} \\
& >\widetilde{h}\left(v_{1}\right)=\widetilde{h}\left(v_{2}\right)=\widehat{d} .
\end{aligned}
$$

This is also a contradiction. Consequently, the distribution of $z\left(\xi_{0}\right)$ and $z\left(\eta_{0}\right)$ only have the following two cases.

Case 1. $z\left(\eta_{0}\right) \leq \ln v_{2} \leq z\left(\xi_{0}\right)$. Noticing that

$$
\begin{aligned}
& z\left(\eta_{0}\right) \geq u_{1}\left(\eta_{1}\right)-u_{2}\left(\xi_{2}\right), \\
& z\left(\xi_{0}\right) \leq u_{1}\left(\xi_{1}\right)-u_{2}\left(\eta_{2}\right),
\end{aligned}
$$

from (27), (30) and (33), we obtain

$$
\begin{aligned}
& u_{2}\left(\xi_{2}\right) \geq u_{1}\left(\eta_{1}\right)-z\left(\eta_{0}\right) \geq u_{1}\left(\eta_{1}\right)-\ln v_{2}>B_{1}-\ln v_{2}, \\
& u_{2}\left(\eta_{2}\right) \leq u_{1}\left(\xi_{1}\right)-z\left(\xi_{0}\right) \leq u_{1}\left(\xi_{1}\right)-\ln v_{2}<B_{2}-\ln v_{2} .
\end{aligned}
$$

By Lemma 2, we find from (26) and (51) that for all $t \in I_{\omega}$

$$
\begin{aligned}
u_{2}(t) & \geq u_{2}\left(\xi_{2}\right)-\frac{1}{2} \int_{\kappa}^{\kappa+\omega}\left|u_{2}^{\Delta}(t)\right| \Delta t \\
& >B_{1}-\ln v_{2}-\frac{\widehat{D}+\widehat{d}}{2} \omega:=B_{7}, \\
u_{2}(t) & \leq u_{2}\left(\eta_{2}\right)+\frac{1}{2} \int_{\kappa}^{\kappa+\omega}\left|u_{2}^{\Delta}(t)\right| \Delta t \\
& <B_{2}-\ln v_{2}+\frac{\widehat{D}+\widehat{d}}{2} \omega:=B_{8} .
\end{aligned}
$$

Case 2. $z\left(\eta_{0}\right) \leq \ln v_{1} \leq z\left(\xi_{0}\right)$. From (27), (30) and (33), we also obtain

$$
\begin{aligned}
& u_{2}\left(\xi_{2}\right) \geq u_{1}\left(\eta_{1}\right)-z\left(\eta_{0}\right) \geq u_{1}\left(\eta_{1}\right)-\ln v_{1}>B_{1}-\ln v_{1}, \\
& u_{2}\left(\eta_{2}\right) \leq u_{1}\left(\xi_{1}\right)-z\left(\xi_{0}\right) \leq u_{1}\left(\xi_{1}\right)-\ln v_{1}<B_{2}-\ln v_{1} .
\end{aligned}
$$

By Lemma 2, we find from (26) and (53) that for all $t \in I_{\omega}$

$$
\begin{aligned}
u_{2}(t) & \geq u_{2}\left(\xi_{2}\right)-\frac{1}{2} \int_{\kappa}^{\kappa+\omega}\left|u_{2}^{\Delta}(t)\right| \Delta t \\
& >B_{1}-\ln v_{1}-\frac{\widehat{D}+\widehat{d}}{2} \omega:=B_{9}, \\
u_{2}(t) & \leq u_{2}\left(\eta_{2}\right)+\frac{1}{2} \int_{\kappa}^{\kappa+\omega}\left|u_{2}^{\Delta}(t)\right| \Delta t \\
& <B_{2}-\ln v_{1}+\frac{\widehat{D}+\widehat{d}}{2} \omega:=B_{10} .
\end{aligned}
$$

By $\left(\mathrm{H}_{9}\right)$, we know that

$$
B_{7}<B_{8}<B_{9}<B_{10} .
$$

Clearly, $B_{7}, B_{8}, B_{9}$, and $B_{10}$ are independent of $\lambda$.

By the monotonicity of functions $\tilde{f}$ and $\widetilde{h},\left(\mathrm{H}_{3}\right),\left(\mathrm{H}_{5}\right)$, and $\left(\mathrm{H}_{6}\right)$, it is easy to show that algebraic system (40) has two distinct solutions:

$$
u^{(i)}=\left(\ln V_{0}^{(i)}, \ln V_{0}^{(i)}-\ln v_{i}\right)^{\mathrm{T}}, \quad i=1,2,
$$

where $V_{0}^{(i)}$ is the unique solution of equation $\tilde{f}(v)-\widetilde{p}\left(v_{i}\right)=0$. Obviously,

$$
V_{1} \leq V_{0}^{(i)}<V_{2}
$$

We now take

$$
\begin{aligned}
& \Omega_{1}=\{\left(u_{1}(t), u_{2}(t)\right)^{\mathrm{T}} \in X:\left|u_{1}(t)\right| \\
&\left.<B_{5}+\beta_{0}, u_{2}(t) \in\left(B_{7}, B_{8}\right)\right\}, \\
& \Omega_{2}=\left\{\left(u_{1}(t), u_{2}(t)\right)^{\mathrm{T}} \in X:\left|u_{1}(t)\right|\right. \\
&\left.<B_{5}+\beta_{0}, u_{2}(t) \in\left(B_{9}, B_{10}\right)\right\},
\end{aligned}
$$


where $\beta_{0}$ is taken sufficiently large such that

$$
\beta_{0}>\max \left\{\left|\ln V_{0}^{(1)}\right|,\left|\ln V_{0}^{(2)}\right|\right\} \text {, }
$$

Then both $\Omega_{1}$ and $\Omega_{2}$ are bounded open subsets of $X$. It follows from (55), (57), and (59) that $u^{(1)} \in \Omega_{1}, u^{(2)} \in \Omega_{2}$, and $\bar{\Omega}_{1} \cap \bar{\Omega}_{2}=\varnothing$. With the help of (38), (52), (54), and (59), it is easy to see that $\Omega_{1}$ and $\Omega_{2}$ satisfy condition (a) in Lemma 1 . When $\left(u_{1}(t), u_{2}(t)\right)^{\mathrm{T}} \in \partial \Omega_{i} \cap \operatorname{Ker} L=\partial \Omega_{i} \cap \mathbb{R}^{2}(i=1,2)$, $\left(u_{1}(t), u_{2}(t)\right)^{\mathrm{T}}$ is a constant vector in $\mathbb{R}^{2}$. Thus, we have

$$
Q N\left(\begin{array}{l}
u_{1} \\
u_{2}
\end{array}\right)=\left(\begin{array}{c}
\tilde{f}\left(\mathrm{e}^{u_{1}}\right)-\widetilde{p}\left(\mathrm{e}^{u_{1}-u_{2}}\right) \\
-\widehat{d}+\widetilde{h}\left(\mathrm{e}^{u_{1}-u_{2}}\right)
\end{array}\right) \neq\left(\begin{array}{l}
0 \\
0
\end{array}\right) .
$$

This proves that condition (b) in Lemma 1 is satisfied. A direct calculation shows that

$$
\begin{aligned}
\operatorname{deg} & \left(J Q N, \Omega_{i} \cap \operatorname{Ker} L, 0\right) \\
& =\operatorname{sign}\left\{-v_{i} V_{0}^{(i)} \tilde{f}^{\prime}\left(V_{0}^{(i)}\right) \tilde{h}^{\prime}\left(v_{i}\right)\right\} \\
& =(-1)^{i-1} \neq 0, \quad i=1,2 .
\end{aligned}
$$

By now we have proved that $\Omega_{1}$ and $\Omega_{2}$ satisfy all the requirements in Lemma 1 . Hence, system (1) has at least two $\omega$-periodic solutions $u^{*}(t)$ and $u^{\dagger}(t)$ in $\operatorname{Dom} L \cap \bar{\Omega}_{1}$ and Dom $L \cap \bar{\Omega}_{2}$, respectively. This completes the proof.

Noticing that both systems (2) and (3) are special cases of system (1), by Theorem 6 , we can obtain the following results.

Theorem 7. Suppose that $\left(H_{1}\right)-\left(H_{6}\right)$ and $\left(H_{8}\right)-\left(H_{9}\right)$ hold. Then system (2) has at least two positive $\omega$-periodic solutions.

Theorem 8. Suppose that $\left(H_{1}\right)-\left(H_{6}\right)$ and $\left(H_{8}\right)-\left(H_{9}\right)$ hold. Then system (3) has at least two positive $\omega$-periodic solutions.

Remark 9. The proof of Theorems 3 and 6 shows that all the above results also remain valid if some delay terms are replaced by terms with distributed or state-dependent delays.

Remark 10. In their Theorem 2.2, Ding and Jiang [14] proved that (2) has at least two positive $\omega$-periodic solutions if $\left(\mathrm{H}_{1}\right)-$ $\left(\mathrm{H}_{6}\right),\left(\mathrm{H}_{8}\right)$, and

$$
\left(\mathrm{H}_{9}^{\prime}\right)\left(V_{2} / V_{1}\right) \mathrm{e}^{2[\widetilde{F}(0)+\tilde{f}(0)+\widehat{D}+\widehat{d}] \omega}<v_{2} / v_{1},
$$

hold. Obviously, the condition $\left(\mathrm{H}_{9}^{\prime}\right)$ implies $\left(\mathrm{H}_{9}\right)$. Hence, our Theorem 7 improves Theorem 2.2 of [14].

Remark 11. Shao [10] studied a special case of system (1) for $f(t, x)=a(t)-b(t) x$. Therefore, our Theorem 3 generalizes Theorem 1 of [10].

Remark 12. Fan and Wang [17], Wang and Li [21], and Fan et al. $[16,18]$ studied some special cases of system (2) for $f(t, x)=a(t)-b(t) x$. Therefore, our Theorem 4 generalizes Theorem 2.1 of [17], Theorem 3.1 of [16], Theorem 3.5 of [18], and Theorem 2.1 of [21].
Remark 13. Fan and Wang [23] and Xia et al. [24] studied some special cases of system (3) for $f(t, x)=a(t)-b(t) x$. Therefore, our Theorem 5 generalizes Theorem 2.1 of [23], and Theorem 3.1 of [24].

\section{Acknowledgments}

This work is supported by the National Natural Science Foundation of China under Grant 11271110, the Key Programs for Science and Technology of the Education Department of Henan Province under Grant 12A110007, and the Scientific Research Start-up Funds of Henan University of Science and Technology.

\section{References}

[1] M. Bohner and A. Peterson, Dynamic Equations on Time Scales: An Introduction with Applications, Birkhäuser, Boston, Mass, USA, 2001.

[2] M. Bohner and A. Peterson, Advances in Dynamic Equations on Time Scales, Birkhäuser, Boston, Mass, USA, 2003.

[3] M. Bohner, L. Erbe, and A. Peterson, "Oscillation for nonlinear second order dynamic equations on a time scale," Journal of Mathematical Analysis and Applications, vol. 301, no. 2, pp. 491507, 2005.

[4] M. Bohner, M. Fan, and J. Zhang, "Existence of periodic solutions in predator-prey and competition dynamic systems," Nonlinear Analysis. Real World Applications, vol. 7, no. 5, pp. 1193-1204, 2006.

[5] X. Ding and G. Zhao, "Periodic solutions for a semi-ratiodependent predator-prey system with delays on time scales," Discrete Dynamics in Nature and Society, vol. 2012, Article ID 928704, 15 pages, 2012.

[6] M. Fazly and M. Hesaaraki, "Periodic solutions for a semiratio-dependent predator-prey dynamical system with a class of functional responses on time scales," Discrete and Continuous Dynamical Systems B, vol. 9, no. 2, pp. 267-279, 2008.

[7] J. Hoffacker and C. C. Tisdell, "Stability and instability for dynamic equations on time scales," Computers \& Mathematics with Applications, vol. 49, no. 9-10, pp. 1327-1334, 2005.

[8] Y. Li and H. Zhang, "Existence of periodic solutions for a periodic mutualism model on time scales," Journal of Mathematical Analysis and Applications, vol. 343, no. 2, pp. 818-825, 2008.

[9] J. Liu, Y. Li, and L. Zhao, "On a periodic predator-prey system with time delays on time scales," Communications in Nonlinear Science and Numerical Simulation, vol. 14, no. 8, pp. 3432-3438, 2009.

[10] Y. Shao, "Existence of positive periodic solution of a ratiodependent predator-prey model on time scale with monotonic functional response," Advances in Mathematics, vol. 39, no. 2, pp. 224-232, 2010.

[11] B. B. Zhang and M. Fan, "A remark on the application of coincidence degree to periodicity of dynamic equtions on time scales," Journal of Northeast Normal University, vol. 39, no. 4, pp. $1-3,2007$.

[12] L. Zhang, H.-X. Li, and X.-B. Zhang, "Periodic solutions of competition Lotka-Volterra dynamic system on time scales," Computers \& Mathematics with Applications, vol. 57, no. 7, pp. 1204-1211, 2009. 
[13] X. Ding, "Positive periodic solutions in generalized ratiodependent predator-prey systems," Nonlinear Analysis. Real World Applications, vol. 9, no. 2, pp. 394-402, 2008.

[14] X. Ding and J. Jiang, "Multiple periodic solutions in delayed Gause-type ratio-dependent predator-prey systems with nonmonotonic numerical responses," Mathematical and Computer Modelling, vol. 47, no. 11-12, pp. 1323-1331, 2008.

[15] Y. H. Fan and W. T. Li, "Permanence in delayed ratio-dependent predator-prey models with monotonic functional responses," Nonlinear Analysis. Real World Applications, vol. 8, no. 2, pp. 424-434, 2007.

[16] Y. H. Fan, W. T. Li, and L. L. Wang, "Periodic solutions of delayed ratio-dependent predator-prey models with monotonic or nonmonotonic functional responses," Nonlinear Analysis. Real World Applications, vol. 5, no. 2, pp. 247-263, 2004.

[17] M. Fan and K. Wang, "Periodicity in a delayed ratio-dependent predator-prey system," Journal of Mathematical Analysis and Applications, vol. 262, no. 1, pp. 179-190, 2001.

[18] M. Fan, Q. Wang, and X. Zou, "Dynamics of a non-autonomous ratio-dependent predator-prey system," Proceedings of the Royal Society of Edinburgh A, vol. 133, no. 1, pp. 97-118, 2003.

[19] S.-B. Hsu, T.-W. Hwang, and Y. Kuang, "Global analysis of the Michaelis-Menten-type ratio-dependent predator-prey system," Journal of Mathematical Biology, vol. 42, no. 6, pp. 489$506,2001$.

[20] Y. Kuang, "Rich dynamics of Gauss-type ratio-dependent predator-prey systems," Fields Institute Communications, vol. 21, pp. 325-337, 1999.

[21] L.-L. Wang and W.-T. Li, "Periodic solutions and permanence for a delayed nonautonomous ratio-dependent predator-prey model with Holling type functional response," Journal of Computational and Applied Mathematics, vol. 162, no. 2, pp. 341-357, 2004.

[22] Y. Xia and M. Han, "Multiple periodic solutions of a ratiodependent predator-prey model," Chaos, Solitons \& Fractals, vol. 39, no. 3, pp. 1100-1108, 2009.

[23] M. Fan and K. Wang, "Periodic solutions of a discrete time nonautonomous ratio-dependent predator-prey system," Mathematical and Computer Modelling, vol. 35, no. 9-10, pp. 951-961, 2002.

[24] Y. Xia, J. Cao, and M. Lin, "Discrete-time analogues of predatorprey models with monotonic or nonmonotonic functional responses," Nonlinear Analysis. Real World Applications, vol. 8, no. 4, pp. 1079-1095, 2007.

[25] R. E. Gaines and J. L. Mawhin, Coincidence Degree, and Nonlinear Differential Equations, vol. 568 of Lecture Notes in Mathematics, Springer, Berlin, Germany, 1977. 


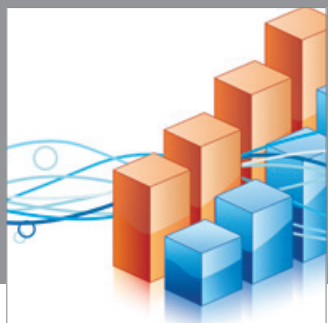

Advances in

Operations Research

mansans

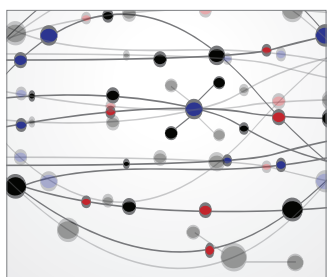

The Scientific World Journal
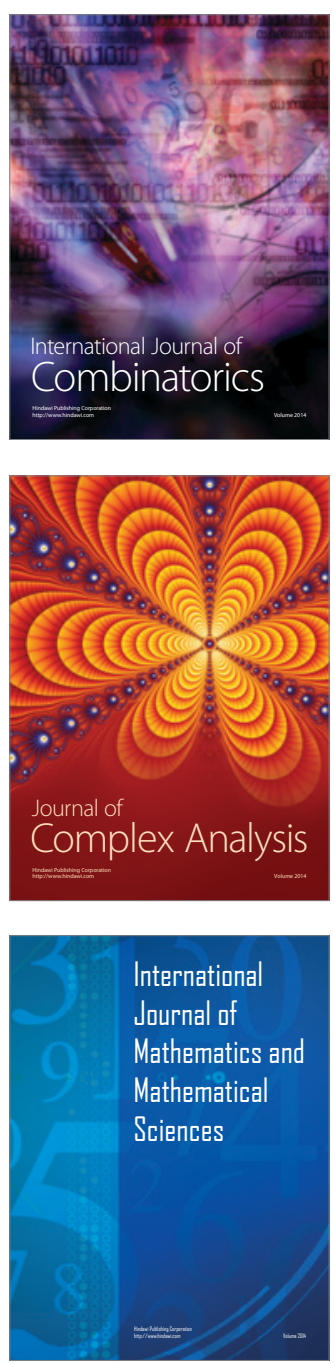
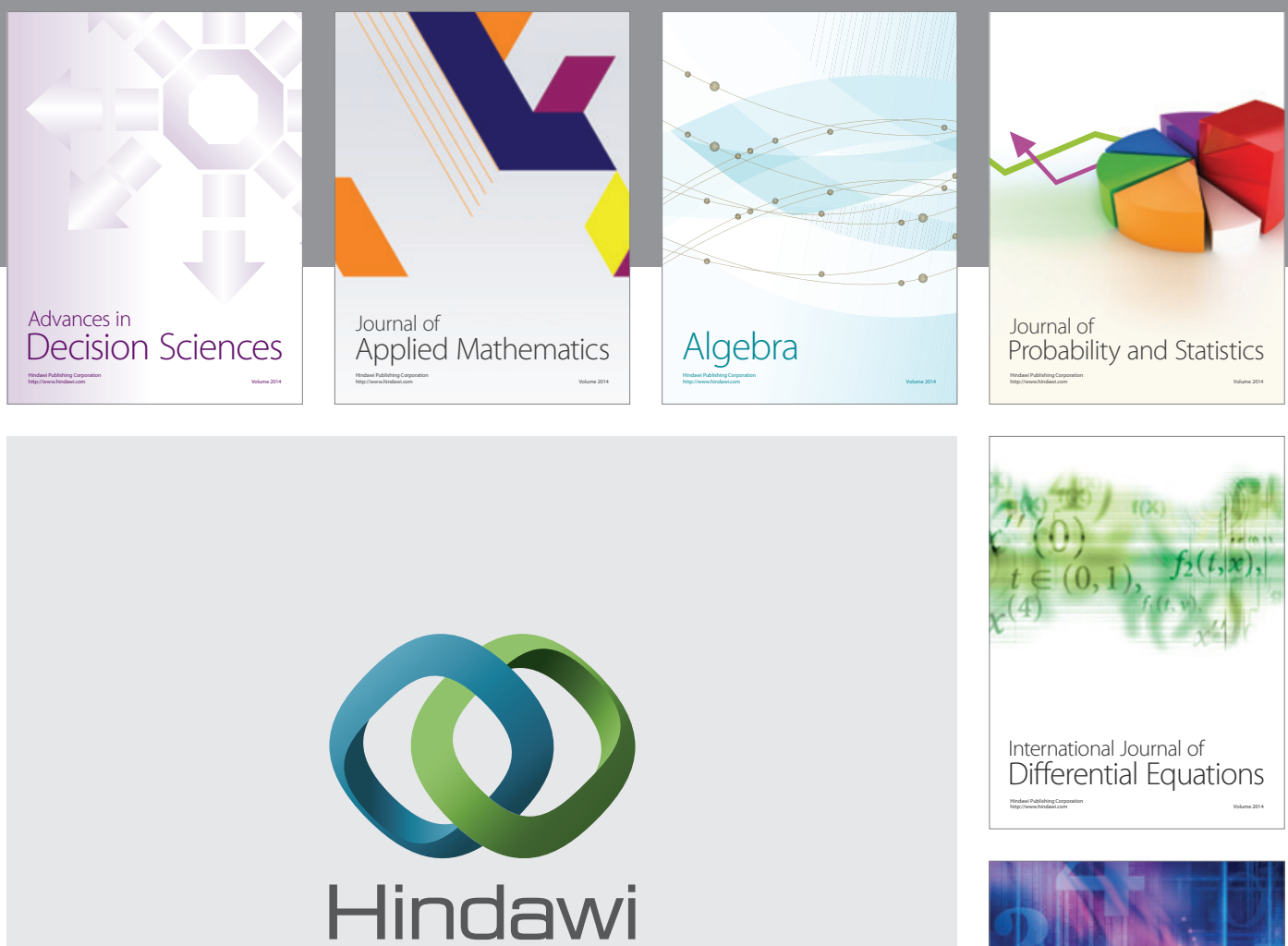

Submit your manuscripts at http://www.hindawi.com
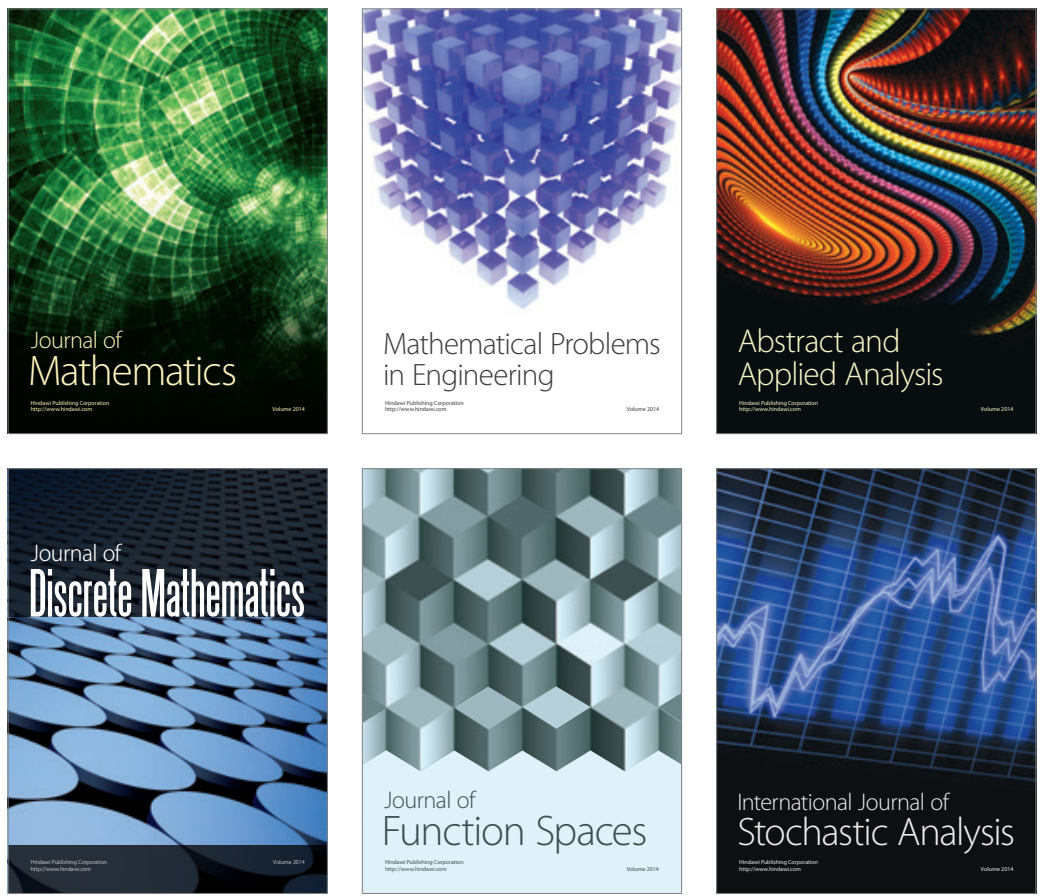

Journal of

Function Spaces

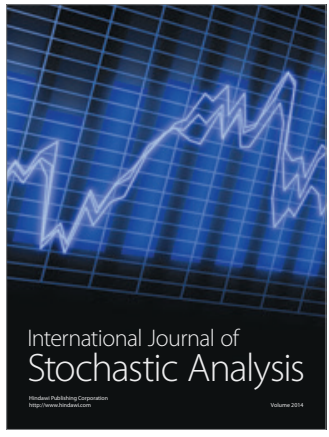

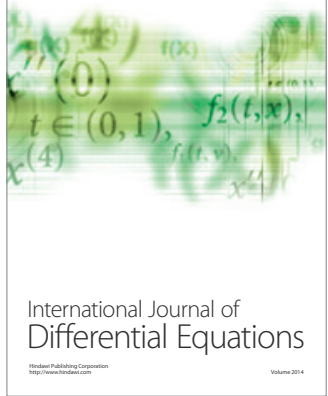
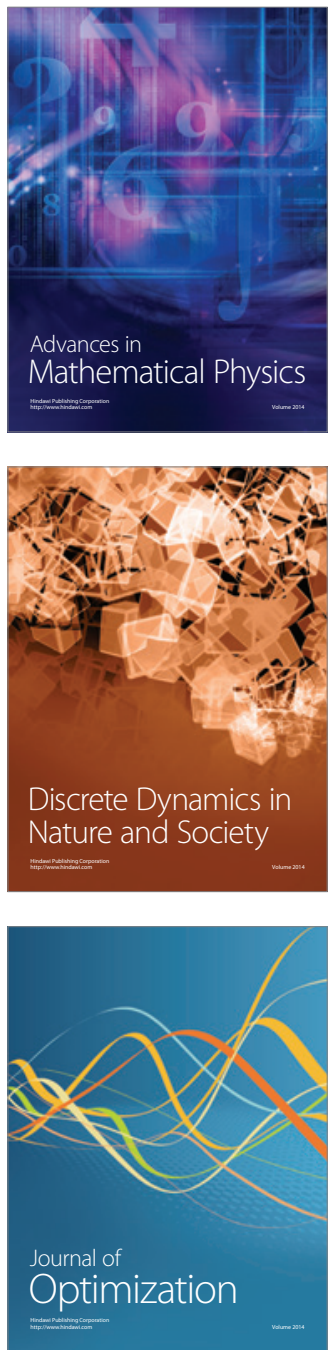